\title{
Mutant knots and intersection graphs
}

\author{
Sergei V Chmutov \\ SERGEI K LANDO
}

\begin{abstract}
We prove that if a finite order knot invariant does not distinguish mutant knots, then the corresponding weight system depends on the intersection graph of a chord diagram rather than on the diagram itself. Conversely, if we have a weight system depending only on the intersection graphs of chord diagrams, then the composition of such a weight system with the Kontsevich invariant determines a knot invariant that does not distinguish mutant knots. Thus, an equivalence between finite order invariants not distinguishing mutants and weight systems depending only on intersections graphs is established. We discuss the relationship between our results and certain Lie algebra weight systems.
\end{abstract}

57M25, 57M15; 57M27, 05C10

\section{Introduction}

Below, we use standard notions of the theory of finite order, or Vassiliev, invariants of knots in 3-space; their definitions can be found, for example, in Chmutov, Duzhin and Mostovoy [6] or Lando and Zvonkin [14], and we recall them briefly in Section 2. All knots are assumed to be oriented.

Two knots are said to be mutant if they differ by a rotation of a tangle with four endpoints about either a vertical axis, a horizontal axis, or an axis perpendicular to the page. If necessary, the orientation inside the tangle may be replaced by the opposite one. Two famous examples of mutant knots are the Conway (11n34) knot $C$ of genus 3 and the Kinoshita-Terasaka (11n42) knot $K T$ of genus 2, drawn in Figure 1 (see The Knot Atlas [1]). Note that the change of the orientation of a knot can be achieved by a mutation in the complement to a trivial tangle.
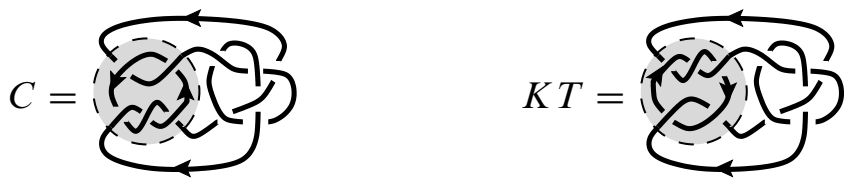

Figure 1: The Conway knot $C$ and the Kinotshita-Terasaka knot $K$ 
Many known knot invariants cannot distinguish mutant knots. Neither the Alexander polynomial, nor the (colored) Jones polynomial, nor the HOMFLY as well as the Kauffman two variable polynomials distinguish mutants; see, for example, Morton and Cromwell [17].

On the other hand, a big class of knot invariants, known as Vassiliev or finite order knot invariants has been thoroughly studied during the last decade. Finite order knot invariants form a filtered commutative associative ring. All Vassiliev invariants up to order 10 do not distinguish mutants as well; see Murakami [18]. However, there is a Vassiliev invariant of order 11 distinguishing $C$ and $K T[17 ; 18]$. It comes from the colored HOMFLY polynomial.

Vassiliev knot invariants can be described in terms of weight systems, that is, functions on chord diagrams, which are combinatorial objects consisting of chords with disjoint ends in a circle, satisfying certain conditions. In the present paper, we give a description of Vassiliev invariants not distinguishing mutants. Namely, we show (Theorem 1) that they are exactly those associated to weight systems whose values depend on the intersection graph of a chord diagram rather than the diagram itself. Distinct chord diagrams can have coinciding intersection graphs, and the vector space of weight systems depending on intersections graphs is smaller than that of all weight systems.

The study of weight systems determined by intersection graphs was initiated by Chmutov, Duzhin and Lando [5], and a number of interesting such invariants has been discovered since then. Our results imply that the weight systems associated to the Lie algebra $\mathfrak{s l}(2)$ and the Lie superalgebra $\mathfrak{g l}(1 \mid 1)$ also belong to this class. These weight systems are the ones corresponding to the colored Jones and Alexander polynomials respectively.

In Section 2, we recall necessary definitions and state the main results of the paper. Section 3 is devoted to the proof of Theorem 1. In Section 4, we discuss the relationship between intersection graphs and the weight systems associated to the Lie algebra $\mathfrak{s l}(2)$ and the Lie superalgebra $\mathfrak{g l}(1 \mid 1)$.

The paper was written during the second author's visit to the Department of Mathematics of Ohio State University. He expresses his gratitude to this institution for warm hospitality and excellent working conditions. We are grateful to S Duzhin, C Soulié, K J Supowit, and A Vaintrob for useful discussions. We are indebted to T Ohtsuki for numerous valuable suggestions on improvement of the exposition and for an alternative proof of Theorem 3. The second author was partly supported by the grant ACI-NIM2004-243 (Noeuds et tresses), RFBR 05-01-01012-a, NWO-RFBR 047.011.2004.026 (RFBR 05-02-89000-NWOa), GIMP ANR-05-BLAN-0029-01. 


\section{Definitions and statements of main results}

Let us recall the notions of Vassiliev invariant and weight system. A knot is a smooth nondegenerate embedding of $S^{1}$ into 3 -space, and a knot invariant is a function on the set of knots $\mathcal{K}$ taking the same values on isotopic knots. If otherwise is not stated explicitly, all knot invariants are assumed to take values in $\mathbb{Q}$. Any knot invariant can be extended to singular knots having only double points according to the Vassiliev skein relation

$$
v(\nearrow)=v(X / \nearrow)-v(\nearrow)
$$

A knot invariant is said to be of order at most $n$ if its extension vanishes on each singular knot with $n+1$ double points. Knot invariants of order at most $n$, for some $n$, are finite order, or Vassiliev, invariants. The value of a knot invariant of order at most $n$ on a singular knot with $n$ double points depends on the chord diagram of the knot, that is, the source circle $S^{1}$ with chords whose ends are the preimages of the double points, rather than on the knot itself. Thus, any knot invariant of order at most $n$ determines a function on chord diagrams with $n$ chords.

Any function $w$ on chord diagrams obtained in this way satisfies the so-called four-term relations,

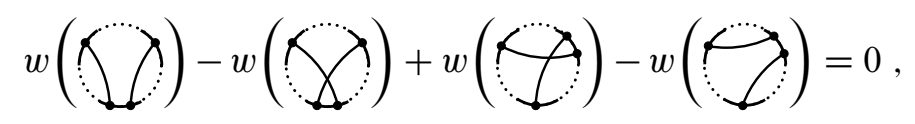

where the dotted arcs of the four diagrams can carry an arbitrary set of chords, the same for all the four pictures. In addition, any such function vanishes on all chord diagrams having an isolated chord, that is, a chord intersecting no other chord (the one-term, or (1T), relations). The Kontsevich theorem [12] states that these are the only restrictions: any function satisfying the four-term and the one-term relations is obtained from a finite order invariant of knots by means of the above procedure. The proof of the theorem is based on a construction known as the Kontsevich integral.

The notion of finite order invariant can be extended to framed knots, that is, knots endowed with a framing, which is a smooth nondegenerate embedding of a tubular neighborhood of the zero section in the tangent bundle to $S^{1}$ to 3 -space. The extension [15] of Kontsevich's theorem to framed knots states that the corresponding functions on chord diagrams are exactly those that satisfy the (4T)-relations. Functions on chord diagrams satisfying the four-term relations are called weight systems. Again, if it is not stated otherwise, weight systems are assumed to take values in $\mathbb{Q}$. Weight systems satisfying, in addition, (1T)-relations are said to be unframed. Weight systems 
form a graded commutative cocommutative Hopf algebra, and unframed weight systems is a Hopf subalgebra in it.

The Kontsevich invariant $K I$, constructed on the base of the Kontsevich integral, is an invariant taking any knot to the Hopf algebra $\mathcal{A}=\mathcal{A}_{0} \oplus \mathcal{A}_{1} \oplus \mathcal{A}_{2} \oplus \ldots$ of chord diagrams over $\mathbb{Q}$, which is dual to that of unframed weight systems. Here $\mathcal{A}_{n}:=\mathbf{A}_{n} /(4 \mathrm{~T}+1 \mathrm{~T})$ is the quotient space of the space $\mathbf{A}_{n}$ spanned by all chord diagrams with $n$ chords modulo the subspace spanned by all quadruples of chord diagrams in the 4T-relations and all chord diagrams having an isolated chord.

Any unframed weight system induces, in composition with the Kontsevich invariant, a finite order invariant of knots. Such knot invariants are called canonical. The Kontsevich integral is universal, in the sense that the canonical invariants span the whole space of Vassiliev invariants.

To a chord diagram, its intersection graph (also called circle graph) is associated. The vertices of the graph correspond to the chords of the diagram, and two vertices are connected by an edge if and only if the corresponding chords intersect. Thus, any function $g$ on graphs determines, through the triangle

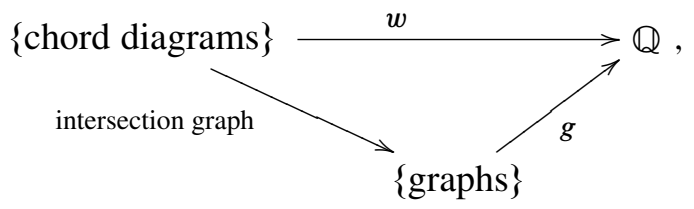

a function $w$ on chord diagrams.

Direct calculations for small $n$ show that the values of weight systems are uniquely determined by the intersection graphs of the chord diagrams. This fact motivated the intersection graph conjecture in [5] (see also [6]) which states that any weight system depends on the intersection graph only. This conjecture happened to be false, because of the existence of a finite order invariant that distinguishes two mutant knots mentioned above and the following fact.

The canonical knot invariant induced by an unframed weight system whose values depend only on the intersection graph of the chord diagrams cannot distinguish mutants.

Our goal is to prove the converse statement thus establishing an equivalence between finite order knot invariants nondistinguishing mutants and unframed weight systems depending on the intersection graphs of chord diagrams only.

Theorem 1 If a finite order knot invariant does not distinguish mutants, then the corresponding unframed weight system depends only on the intersection graphs of chord diagrams. 
Together, the two statements can be combined as follows.

A canonical knot invariant does not distinguish mutants if and only if its weight system depends on the intersection graphs of chord diagrams only.

The same statement is true about finite order invariants of framed knots and arbitrary weight systems.

Here is the diagram of relevant spaces and maps between them.

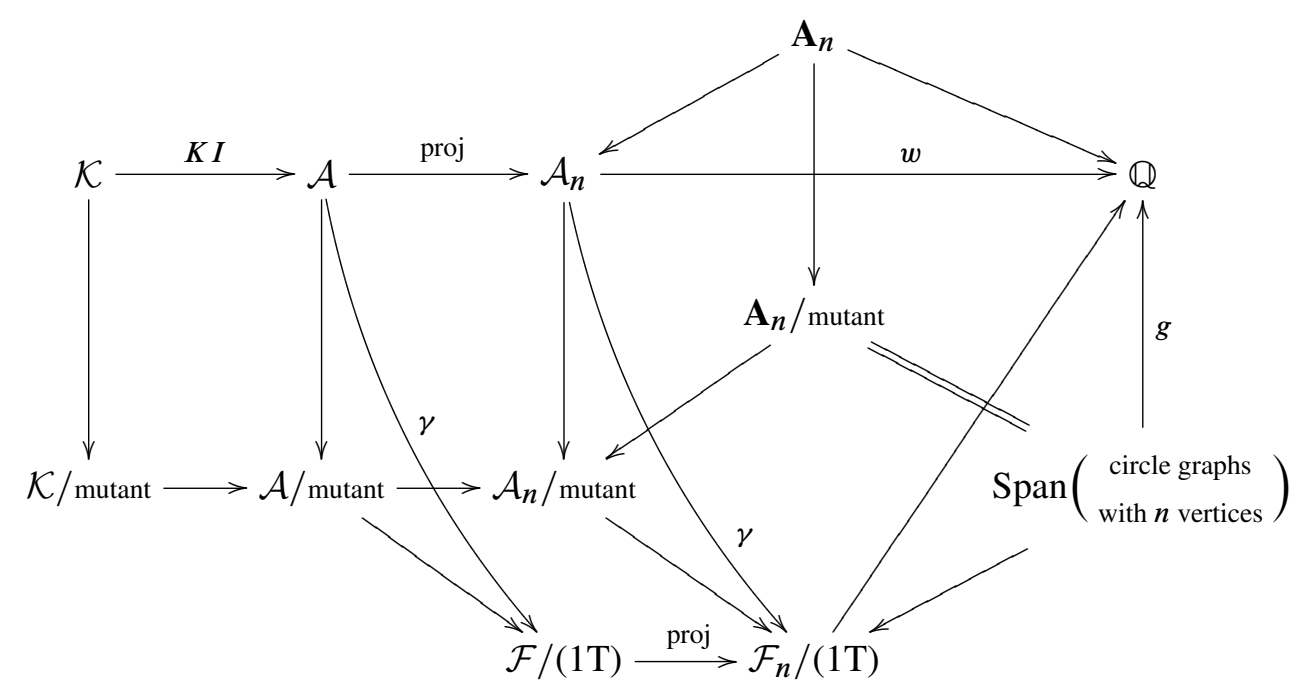

The equivalence relation "mutant" on chord diagrams will be defined in Section 3.1. The equality $\mathbf{A}_{n} /$ mutant $=\operatorname{Span}$ (circle graphs with $n$ vertices) is the result of Theorem 2 there. The space $\mathcal{F}_{n}$ is the degree $n$ part of the 4-bialgebra of graphs $\mathcal{F}$ introduced in [13] (see Section 4.2), and the (1T)-relations in $\mathcal{F}$ are defined as spanned by graphs with isolated vertices.

Recently, B Mellor [16] extended the concept of intersection graph to string links. We do not know whether our Theorem 1 admits an appropriate generalization.

\section{Proof}

\subsection{Representability of graphs as the intersection graphs of chord dia- grams}

Not every graph can be represented as the intersection graph of a chord diagram. For example, the following graphs are not intersection graphs.

Algebraic ${ }^{3} \mathcal{G}$ Geometric Topology, Volume 7 (2007) 

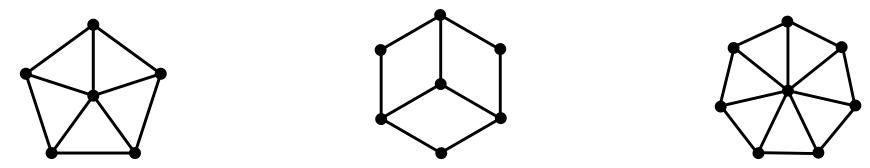

A characterization of those graphs that can be realized as intersection graphs is given by an elegant theorem of A Bouchet [4].

On the other hand, different diagrams may have coinciding intersection graphs. For example, next three diagrams have the same intersection graph
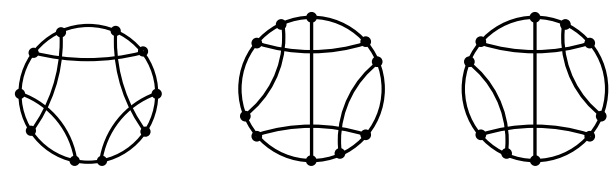

A combinatorial analog of the tangle in mutant knots is a share [5; 6]. Informally, a share of a chord diagram is a subset of chords whose endpoints are separated into at most two parts by the endpoints of the complementary chords. More formally, we have the following:

Definition 1 A share in a chord diagram is a union of two arcs of the outer circle and chords ending on them possessing the following property: each chord one of whose ends belongs to these arcs has both ends on these arcs.

Here are some examples:
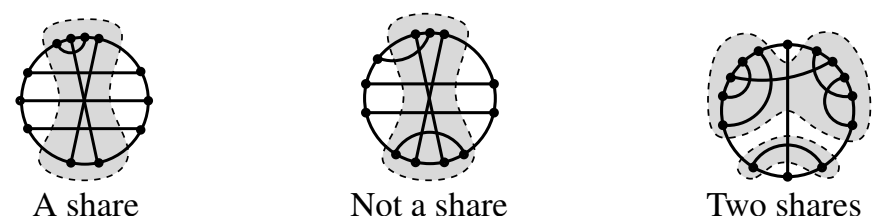

The complement of a share also is a share. The whole chord diagram is its own share whose complement contains no chords.

Definition 2 A mutation of a chord diagram is another chord diagram obtained by a rotation of a share about one of the three axes. Two chord diagrams are said to be mutant if they can be transformed into one another by a sequence of mutations.

For example, three mutations of the share in the first chord diagram above produce the mutations in Figure 2.

Obviously, mutations preserve the intersection graphs of chord diagrams.

Mutations of chord diagram were used in Soulié [19] for studying mutations of alternating links. 

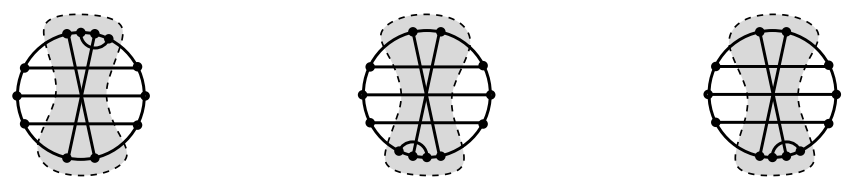

Figure 2

Theorem 2 Two chord diagrams have the same intersection graph if and only if they are related by a sequence of mutations.

This theorem is contained implicitly in Bouchet [3], Courcelle [8] and Gabor, Supowit and Hsu [11] where chord diagrams are written as double occurrence words, the language better suitable for describing algorithms than for topological explanation.

Proof of Theorem 2 The proof of this theorem uses Cunningham's theory of graph decompositions [9].

A split of a (simple) graph $\Gamma$ is a disjoint bipartition $\left\{V_{1}, V_{2}\right\}$ of its set of vertices $V(\Gamma)$ such that each part contains at least 2 vertices, and there are subsets $W_{1} \subseteq V_{1}$, $W_{2} \subseteq V_{2}$ such that all the edges of $\Gamma$ connecting $V_{1}$ with $V_{2}$ form the complete bipartite graph $K\left(W_{1}, W_{2}\right)$ with the parts $W_{1}$ and $W_{2}$. Thus for a split $\left\{V_{1}, V_{2}\right\}$ the whole graph $\Gamma$ can be represented as a union of the induced subgraphs $\Gamma\left(V_{1}\right)$ and $\Gamma\left(V_{2}\right)$ linked by a complete bipartite graph.

Another way to think about splits, which is sometimes more convenient and which we shall use in the pictures below, looks like follows. Consider two graphs $\Gamma_{1}$ and $\Gamma_{2}$ each having a distinguished vertex $v_{1} \in V\left(\Gamma_{1}\right)$ and $v_{2} \in V\left(\Gamma_{2}\right)$, respectively, called markers. Construct the new graph $\Gamma=\Gamma_{1} \otimes_{\left(v_{1}, v_{2}\right)} \Gamma_{2}$ whose set of vertices is

$$
V(\Gamma)=\left\{V\left(\Gamma_{1}\right)-v_{1}\right\} \sqcup\left\{V\left(\Gamma_{2}\right)-v_{2}\right\}
$$

and whose set of edges is

$$
\begin{aligned}
E(\Gamma)=\left\{\left(v_{1}^{\prime}, v_{1}^{\prime \prime}\right) \in E\left(\Gamma_{1}\right): v_{1}^{\prime}\right. & \left.\neq v_{1} \neq v_{1}^{\prime \prime}\right\} \sqcup\left\{\left(v_{2}^{\prime}, v_{2}^{\prime \prime}\right) \in E\left(\Gamma_{2}\right): v_{2}^{\prime} \neq v_{2} \neq v_{2}^{\prime \prime}\right\} \\
& \sqcup\left\{\left(v_{1}^{\prime}, v_{2}^{\prime}\right):\left(v_{1}^{\prime}, v_{1}\right) \in E\left(\Gamma_{1}\right) \text { and }\left(v_{2}, v_{2}^{\prime}\right) \in E\left(\Gamma_{2}\right)\right\} .
\end{aligned}
$$

Representation of $\Gamma$ as $\Gamma_{1} \otimes_{\left(v_{1}, v_{2}\right)} \Gamma_{2}$ is called a decomposition of $\Gamma, \Gamma_{1}$ and $\Gamma_{2}$ are called the components of the decomposition. The partition $\left\{V\left(\Gamma_{1}\right)-v_{1}, V\left(\Gamma_{2}\right)-v_{2}\right\}$ is a split of $\Gamma$. Graphs $\Gamma_{1}$ and $\Gamma_{2}$ might be decomposed further giving a finer decomposition of the initial graph $\Gamma$. Pictorially, we represent a decomposition by pictures of its components where the corresponding markers are connected by a dashed edge. 
A prime graph is a graph with at least three vertices admitting no splits. A decomposition of a graph is said to be canonical if the following conditions are satisfied:

(i) Each component is either a prime graph, or a complete graph $K_{n}$, or a star $S_{n}$, which is the tree with a vertex, the center, adjacent to $n$ other vertices.

(ii) No two components that are complete graphs are neighbors, that is, their markers are not connected by a dashed edge.

(iii) The markers of two components that are star graphs connected by a dashed edge are either both centers or both not centers of their components.

W H Cunningham proved [9, Theorem 3] that each graph with at least six vertices possesses a unique canonical decomposition.

Let us illustrate the notions introduced above by two examples of canonical decomposition of the intersection graphs of chord diagrams. We number the chords and the corresponding vertices in our graphs, so that the unnumbered vertices are the markers of the components. The first example is our example from Figure 2:

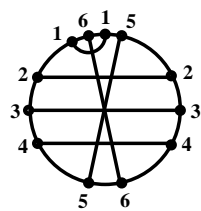

A chord diagram

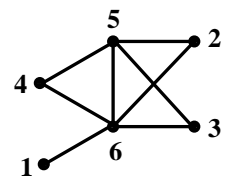

The intersection graph

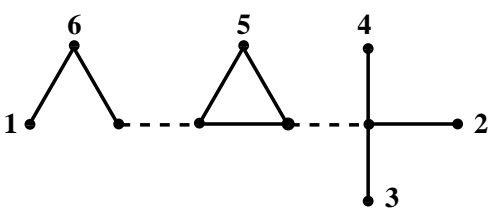

The canonical decomposition

The second example represents the chord diagram of the double points in the plane diagram of the Conway knot $C$ from Figure 1. The double points of the shaded tangle are represented by the chords $1,2,9,10,11$.

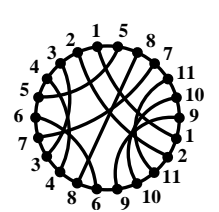

Chord diagram

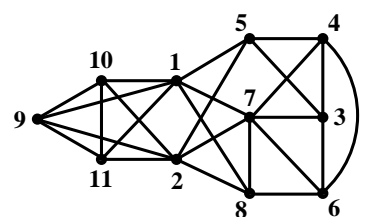

Intersection graph

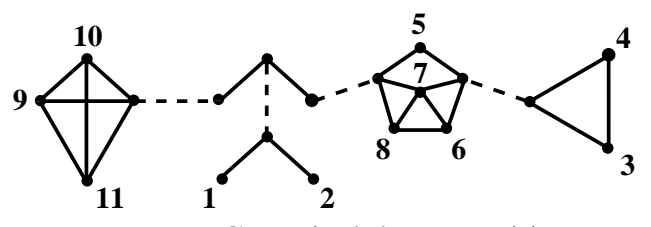

Canonical decomposition

The key observation in the proof of Theorem 2 is that components of the canonical decomposition of any intersection graph admit a unique representation by chord diagrams. For a complete graph and star components, this is obvious. For a prime component, this was proved by A Bouchet [3, Statement 4.4] (see also Gabor, Supowit and Hsu [11, Section 6] for an algorithm finding such a representation for a prime graph).

Algebraic $8 \mathcal{G}$ Geometric Topology, Volume 7 (2007) 
Now to describe all chord diagrams with a given intersection graph, we start with a component of its canonical decomposition. There is only one way to realize the component by a chord diagram. We draw the chord corresponding to the marker as a dashed chord and call it the marked chord. This chord indicates the places where we must cut the circle removing the marked chord together with small arcs containing its endpoints. As a result we obtain a chord diagram on two arcs. Repeating the same procedure with a neighbor component of the canonical decomposition, we get another chord diagram on two arcs. We have to sew these two diagrams together by their arcs in an alternating order. There are four possibilities to do this, and they differ by mutations of the share corresponding to the second (or, alternatively, the first) component. This completes the proof of Theorem 2.

To illustrate the last stage of the proof consider our standard example and take the star 2-3-4 component first and then the triangle component. We get

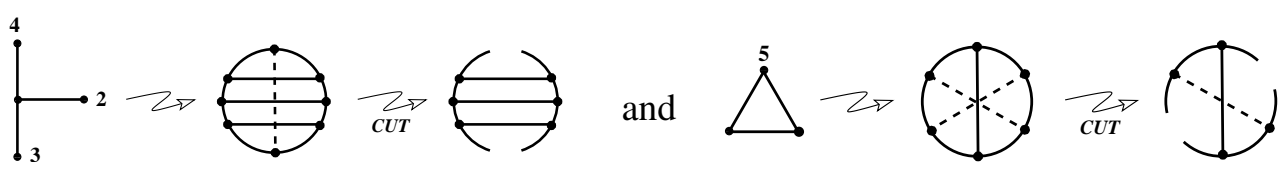

Because of the symmetry, the four ways of sewing these diagrams produce only two distinct chord diagrams with a marked chord:

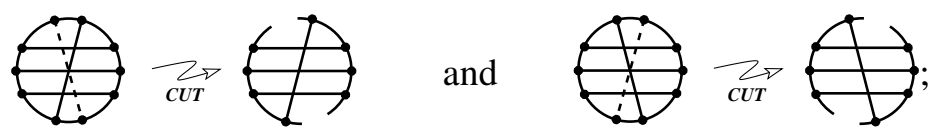

repeating the same procedure with the marked chord for the last 1-6 component of the canonical decomposition, we get

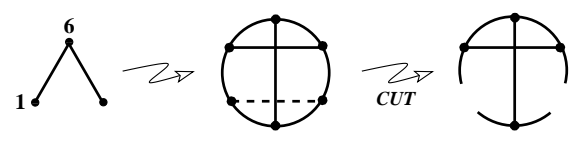

Sewing this diagram into the previous two in all possible ways we get four mutant chord diagrams from Figure 2.

As an enjoyable exercise we leave to the reader to work out our second example with the chord diagram of the diagram of the Conway knot and find the mutation producing the chord diagram of the plane diagram of the Kinoshita-Terasaka knot using the canonical decomposition. 


\subsection{Proof of Theorem 1}

Suppose we have a Vassiliev knot invariant $v$ of order at most $n$ that does not distinguish mutant knots. Let $D_{1}$ and $D_{2}$ be chord diagrams with $n$ chords whose intersection graphs coincide. We are going to prove that the values of the weight system of $v$ on $D_{1}$ and $D_{2}$ are equal.

By Theorem 2, it is enough to consider the case when $D_{1}$ and $D_{2}$ differ by a single mutation in a share $S$. Let $K_{1}$ be a singular knot with $n$ double points whose chord diagram is $D_{1}$. Consider the collection of double points of $K_{1}$ corresponding to the chords occurring in the share $S$. By the definition of a share, $K_{1}$ has two arcs containing all these double points and no others. By sliding the double points along one of these arcs and shrinking the other arc we may enclose these arcs into a ball whose interior does not intersect the rest of the knot. In other words, we may isotope the knot $K_{1}$ to a singular knot so as to collect all the double points corresponding to $S$ in a tangle $T_{S}$. Performing an appropriate rotation of $T_{S}$ we obtain a singular knot $K_{2}$ with the chord diagram $D_{2}$. Since $v$ does not distinguish mutants, its values on $K_{1}$ and $K_{2}$ are equal. Theorem 1 is proved.

To illustrate the proof, let $D_{1}$ be the chord diagram from our standard example. Pick a singular knot representing $D_{1}$, say
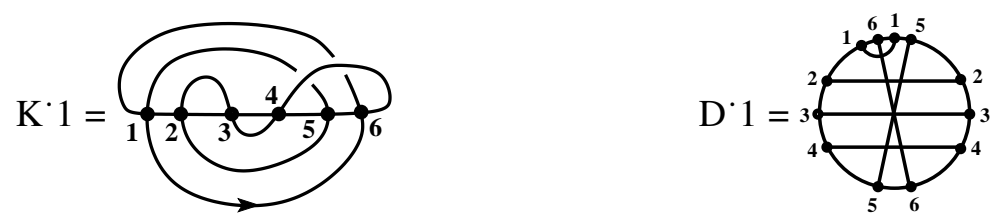

To perform a mutation in the share containing the chords $1,5,6$, we must slide the double point 1 close to the double points 5 and 6 , and then shrink the corresponding arcs:

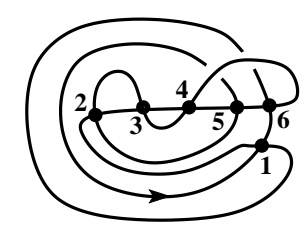

Sliding the double point 1

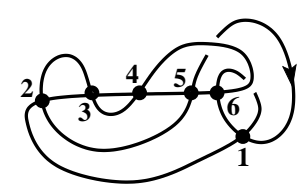

Shrinking the arcs

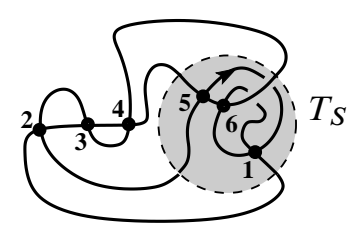

Forming the tangle $T_{S}$

Now doing an appropriate rotation of the tangle $T_{S}$ we obtain a singular knot $K_{2}$ representing the chord diagram $D_{2}$. 


\section{Lie algebra weight systems and intersection graphs}

Kontsevich [12] generalized a construction of Bar-Natan [2] of weight systems defined by a Lie algebra and its representation to a universal weight system, with values in the universal enveloping algebra of the Lie algebra. The weight system associated to a Lie algebra $\mathfrak{g}$ (with a specific invariant scalar product, which we do not mention in notation) is a mapping $W_{\mathfrak{g}}: \mathbf{A}_{n} /(4 \mathrm{~T}) \rightarrow U(\mathfrak{g})^{\mathfrak{g}}$ from the Hopf algebra of chord diagrams to the subalgebra of $\mathfrak{g}$-invariant elements (that is, the center) in the universal enveloping algebra $U(\mathfrak{g})$ of $\mathfrak{g}$. Note that this weight system does not satisfy the one-term relation. In [20], Vaintrob extended this construction to Lie superalgebras.

Any representation $\rho: \mathfrak{g} \rightarrow \mathfrak{g l}(V)$ of a Lie algebra $\mathfrak{g}$ can be extended to a representation of the universal enveloping algebra of $\mathfrak{g}$; we denote this representation by the same letter $\rho$. By taking the trace $\operatorname{Tr}$, this representation determines a number-valued weight system $\operatorname{Tr} \circ \rho \circ W_{\mathfrak{g}}$. Thus, all weight systems associated to representations of a Lie algebra are encoded in the universal weight system. Weight systems associated to representations are said to be colored by the representations.

Our main goal in this section is to prove the following:

Theorem 3 The universal weight systems associated to the Lie algebra sl(2) and to the Lie superalgebra $\mathfrak{g l}(1 \mid 1)$ depend on the intersection graphs of chord diagrams rather than on the diagrams themselves.

Thus, we have the commutative diagram

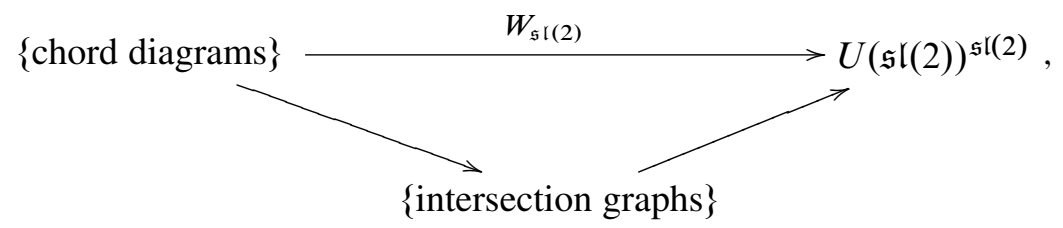

and similarly for $W_{\mathfrak{g l}(1 \mid 1)}$. It follows immediately that the canonical knot invariants corresponding to these two algebras do not distinguish mutants. For $\mathfrak{s l}(2)$, this fact is already known, since this weight system is the one of the colored Jones polynomial; nevertheless, we give a direct proof on the intersection graphs side.

Note that for more complicated Lie algebras the statement of Theorem 3 is no longer true. For example, the universal $\mathfrak{s l}(3)$ weight system distinguishes between the Conway and the Kinoshita-Terasaka knots.

In fact, for each of the two algebras we prove more subtle statements. 
Theorem 4 The universal weight system $W_{\mathfrak{s l}(2)}$ depends on the matroid of the intersection graph of a chord diagram rather than on the intersection graph itself.

This theorem inevitably leads to numerous questions concerning relationship between weight systems and matroid theory, which specialists in this theory may find worth being investigated.

Weight systems have a graph counterpart, so-called 4-invariants of graphs [13]. These are linear functions on the 4 -bialgebra $\mathcal{F}$ of graphs, which is a graph counterpart of chord diagrams. The knowledge that a weight system depends only on the intersection graphs does not guarantee, however, that it arises from a 4-invariant. In particular, we do not know, whether this is true for the universal $\mathfrak{s l}(2)$ weight system. Either positive (with an explicit description) or negative answer to this question would be extremely interesting. For $\mathfrak{g l}(1 \mid 1)$, the answer is positive.

Theorem 5 The universal weight system $W_{\mathfrak{g l}(1 \mid 1)}$ is induced by a 4 -invariant of graphs.

Thus, for $W_{\mathfrak{g r}(1 \mid 1)}$, the commutative triangle acquires the form:

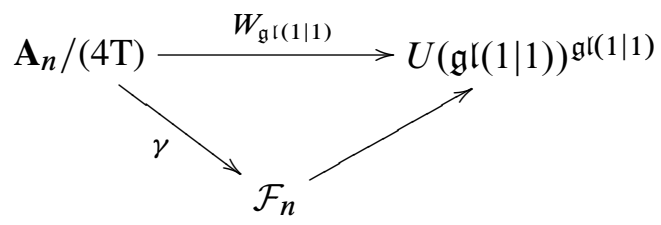

For small orders, the fact that $W_{\mathfrak{g r}(1 \mid 1)}$ depends on intersection graphs only was established in an undergraduate thesis work of David Jordan at the University of Oregon.

In the first two subsections below, we recall the construction of universal weight systems associated to Lie algebras and the notion of 4-invariant of graphs. The next two subsections are devoted to separate treating of the Lie algebra $\mathfrak{s l}(2)$ and the Lie superalgebra $\mathfrak{g l}(1 \mid 1)$ universal weight systems. The last subsection contains Ohtsuki's proof of Theorem 3.

\subsection{Weight systems via Lie algebras}

Our approach follows that of Kontsevich in [12]. In order to construct a weight system, we need a complex Lie algebra $\mathfrak{g}$ endowed with a nondegenerate invariant bilinear form $(\cdot, \cdot)$. The invariance requirement means that $(x,[y, z])=([x, y], z)$ for any three elements $x, y, z \in \mathfrak{g}$. Pick an orthonormal basis $a_{1}, \ldots, a_{d},\left(a_{i}, a_{j}\right)=\delta_{i j}, d$ being the dimension of $\mathfrak{g}$. Any chord diagram can be made into an arc diagram by cutting 
the circle at some point and further straightening it. For an arc diagram of $n$ arcs, write on each arc an index $i$ between 1 and $d$, and then write on both ends of the arc the letter $a_{i}$. Reading all the letters left to right we obtain a word of length $2 n$ in the alphabet $a_{1}, \ldots, a_{d}$, which is an element of the universal enveloping algebra of our Lie algebra. The sum of all these words over all possible settings of the indexes is the element of the universal enveloping algebra $U(\mathfrak{g})$ assigned to the chord diagram. This element is independent of the choice of the cutting point of the circle, as well as the orthonormal basis. It belongs to the center $U(\mathfrak{g})^{\mathfrak{g}}$ of the universal enveloping algebra and satisfies the 4-term relation, whence can be extended to a weight system. The latter is called the universal weight system associated to the Lie algebra and the bilinear form, and is denoted by $W_{\mathfrak{g}}$; it can be specialized to specific representations of the Lie algebra as in the original Bar-Natan's approach. Obviously, any universal weight system is multiplicative: its value on a product of chord diagrams coincides with the product of its values on the factors.

The simplest noncommutative Lie algebra with a nondegenerate invariant bilinear form is $\mathfrak{s l}(2)$. It is 3 -dimensional, and the center $U(\mathfrak{s l}(2))^{\mathfrak{s l}(2)}$ of its universal enveloping algebra is the ring $\mathbb{C}[c]$ of polynomials in a single variable $c$, the Casimir element. The corresponding universal weight system was studied in detail in Chmutov and Varchenko [7]. It attracts a lot of interest because of its equivalence to the colored Jones polynomials.

In [20], Vaintrob generalized Kontsevich's construction to Lie superalgebras, and this construction was elaborated in Figueroa-O'Farrill, Kimura and Vaintrob [10] for the simplest noncommutative Lie superalgebra $\mathfrak{g l}(1 \mid 1)$. The center $U(\mathfrak{g l}(1 \mid 1))^{\mathfrak{g l}(1 \mid 1)}$ of the universal enveloping algebra of this algebra is the ring of polynomials $\mathbb{C}[c, y]$ in two variables. The value of the corresponding universal weight system on a chord diagram with $n$ chords is a quasihomogeneous polynomial in $c$ and $y$, of degree $n$, where the weight of $c$ is set to be 1 , and the weight of $y$ is set to be 2 .

\subsection{The 4-bialgebra of graphs}

By a graph, we mean a finite undirected graph without loops and multiple edges. Let $\mathcal{G}_{n}$ denote the vector space freely spanned over $\mathbb{C}$ by all graphs with $n$ vertices, $\mathcal{G}_{0}=\mathbb{C}$ being spanned by the empty graph. The direct sum

$$
\mathcal{G}=\mathcal{G}_{0} \oplus \mathcal{G}_{1} \oplus \mathcal{G}_{2} \oplus \ldots
$$

carries a natural structure of a commutative cocommutative graded Hopf algebra. The multiplication in this Hopf algebra is induced by the disjoint union of graphs, and the comultiplication is induced by the operation taking a graph $G$ into the sum $\sum G_{U} \otimes G_{\bar{U}}$, 
where $U$ is an arbitrary subset of vertices of $G, \bar{U}$ its complement, and $G_{U}$ denotes the subgraph of $G$ induced by $U$.

The 4-term relation for graphs is defined in the following way. By definition, the 4-term element in $\mathcal{G}_{n}$ determined by a graph $G$ with $n$ vertices and an ordered pair $A, B$ of its vertices is the linear combination

$$
G-G_{A B}^{\prime}-\widetilde{G}_{A B}+\widetilde{G}_{A B}^{\prime},
$$

where

- $G_{A B}^{\prime}$ is the graph obtained by deleting the edge $A B$ in $G$;

- $\widetilde{G}_{A B}$ is the graph obtained by switching the adjacency to $A$ of all the vertices adjacent to $B$ in $G$;

- $\widetilde{G}_{A B}^{\prime}$ is the graph obtained by deleting the edge $A B$ in $G_{A B}^{\prime}$ (or, equivalently, by switching the adjacency to $A$ of all the vertices adjacent to $B$ in $G_{A B}^{\prime}$ ).

All the four terms in a 4-term element have the same number $n$ of vertices. The quotient of $\mathcal{G}_{n}$ modulo the span of all 4 -term elements in $\mathcal{G}_{n}$ (defined by all graphs and all ordered pairs of adjacent vertices in each graph) is denoted by $\mathcal{F}_{n}$. The direct sum

$$
\mathcal{F}=\mathcal{F}_{0} \oplus \mathcal{F}_{1} \oplus \mathcal{F}_{2} \oplus \ldots
$$

is the quotient Hopf algebra of graphs, called the 4-bialgebra. The mapping taking a chord diagram to its intersection graph extends to a graded Hopf algebra homomorphism $\gamma: \mathcal{A} \rightarrow \mathcal{F}$ from the Hopf algebra of chord diagrams to the Hopf algebra of graphs.

Being commutative and cocommutative, the 4-bialgebra is isomorphic to the polynomial ring in its basic primitive elements, that is, it is the tensor product $S\left(\mathcal{P}_{1}\right) \otimes$ $S\left(\mathcal{P}_{2}\right) \otimes \ldots$ of the symmetric algebras of its homogeneous primitive spaces.

\subsection{The $\mathfrak{s l}(2)$ weight system}

Our treatment of the universal weight system associated with the Lie algebra $\mathfrak{s l}(2)$ is based on the recurrence formula for computing the value of this weight system on chord diagrams due to Chmutov and Varchenko [7]. The recurrence states that if a chord diagram contains a leaf, that is, a chord intersecting only one other chord, then the value of $W_{\mathfrak{s l}(2)}$ on the diagram is $(c-1 / 2)$ times its value on the result of deleting the leaf, and, in addition,

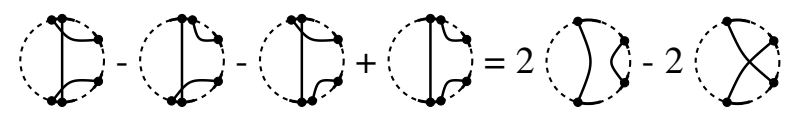

Algebraic $8 \mathcal{G}$ Geometric Topology, Volume 7 (2007) 
meaning that the value of $W_{\mathfrak{s l}(2)}$ on the chord diagram on the left-hand side coincides with the linear combinations of its values on the chord diagrams indicated on the right.

Proof of Theorem 3 Now, in order to prove Theorem 3 for the universal $\mathfrak{s l}$ (2) weight system, we must prove that mutations of a chord diagram preserve the values of this weight system. Take a chord diagram and a share in it. Apply the above recurrence formula to a chord and two its neighbors belonging to the chosen share. The recurrence relation does not affect the complementary share, while all the instances of the modified first share are simpler than the initial one (each of them contains either fewer chords or the same number of chords but with fewer intersections). Repeating this process, we can replace the original share by a linear combination of the simplest shares, chains, which are symmetric meaning that they remain unchanged under rotations. The $\mathfrak{s l}(2)$ case of Theorem 3 is proved.

Proof of Theorem 4 Now let us turn to the proof of Theorem 4. For elementary notions of matroid theory we refer the reader to any standard reference, say to Welsh [21]. Recall that a matroid can be associated to any graph. It is easy to check that the matroid associated to the disjoint union of two graphs coincides with that for the graph obtained by identifying a vertex in the first graph with a vertex in the second one. We call the result of gluing a vertex in a graph $G_{1}$ to a vertex in a graph $G_{2}$ a 1 -product of $G_{1}$ and $G_{2}$. The converse operation is 1-deletion. Of course, the 1-product depends on the choice of the vertices in each of the factors, but the corresponding matroid is independent of this choice.

Similarly, let $G_{1}, G_{2}$ be two graphs, and pick vertices $u_{1}, v_{1}$ in $G_{1}$ and $u_{2}, v_{2}$ in $G_{2}$. Then the matroid associated to the graph obtained by identifying $u_{1}$ with $u_{2}$ and $v_{1}$ with $v_{2}$ coincides with the one associated to the graph obtained by identifying $u_{1}$ with $v_{2}$ and $u_{2}$ with $v_{1}$. The operation taking the result of the first identification to that of the second one is called the Whitney twist on graphs.

Both the 1-product and the Whitney twist have chord diagram analogs. For two chord diagrams with a distinguished chord in each of them, we define their 1-product as a chord diagram obtained by replacing the distinguished chords in the ordinary product of chord diagrams chosen so as to make them neighbors by a single chord connecting their other ends. The Whitney twist also is well defined because of the following statement.

Lemma 1 Suppose the intersection graph of a chord diagram is the result of identifying two pairs of vertices in two graphs $G_{1}$ and $G_{2}$. Then both graphs $G_{1}$ and $G_{2}$ are intersection graphs, as well as the Whitney twist of the original graph. 
The assertion concerning the graphs $G_{1}$ and $G_{2}$ is obvious. In order to prove that the result of the Whitney twist also is an intersection graph, let $c_{1}, c_{2}$ denote the two chords in a chord diagram $C$ such that deleting these chords makes $C$ into an ordinary product of two chord diagrams $C_{1}, C_{2}$. By reflecting the diagram $C_{2}$ and restoring the chords $c_{1}$ and $c_{2}$ we obtain a chord diagram whose intersection graph is the result of the desired Whitney twist. The lemma is proved.

According to the Whitney theorem, two graphs have the same matroid if and only if they can be obtained from one another by a sequence of 1-products/deletions and Whitney twists. Therefore, Theorem 4 follows from:

Lemma 2 (i) The value of $W_{\mathfrak{s l}(2)}$ on the 1-product of chord diagrams coincides with the product of its values on the factors divided by $c$.

(ii) The value of $W_{\mathfrak{s l}(2)}$ remains unchanged under the Whitney twist of the chord diagram.

Statement (i) is proved in [7]. The proof of statement (ii) is similar to that of Theorem 3. Consider the part $C_{2}$ participating in the Whitney twist and apply to it the recurrence relations. Note that the relations do not affect the complementary diagram $C_{1}$. Simplifying the part $C_{2}$ we reduce it to a linear combination of the simplest possible diagrams, chains, which are symmetric under reflection. Reflecting a chain preserves the chord diagram, whence the value of the $\mathfrak{s l}(2)$ weight system. Theorem 4 is proved.

\subsection{The $\mathfrak{g l}(1 \mid 1)$ weight system}

Define the (unframed) Conway graph invariant with values in the ring of polynomials $\mathbb{C}[y]$ in one variable $y$ in the following way. We set it equal to $(-y)^{n / 2}$ on graphs with $n$ vertices if the adjacency matrix of the graph is nondegenerate, and 0 otherwise. Recall that the adjacency matrix $A_{G}$ of a graph $G$ with $n$ vertices is an $(n \times n)$-matrix with entries in $\mathbb{Z}_{2}$ obtained as follows. We choose an arbitrary numbering of the vertices of the graph, and the entry $a_{i j}$ is 1 provided the $i$-th and the $j$-th vertices are adjacent and 0 otherwise (diagonal elements $a_{i i}$ are 0 ). Note that for odd $n$, the adjacency matrix cannot be nondegenerate, hence the values indeed are in the ring of polynomials. The Conway graph invariant is multiplicative: its value on the disjoint union of graphs is the product of its values on the factors.

Clearly, the Conway graph invariant is a 4-invariant. Moreover, it satisfies the 2-term relation, which is more restrictive than the 4-term one: its values on the graphs $G$ and $\widetilde{G}_{A B}$ coincide for any graph $G$ and any pair of ordered vertices $A, B$ in it. Indeed, consider the graph as a symmetric bilinear form on the $\mathbb{Z}_{2}-$ vector space whose basis is 
the set of vertices of the graph, the adjacency matrix being the matrix of the bilinear form in this basis. In these terms, the transformation $G \mapsto \widetilde{G}_{A B}$ preserves the vector space and the bilinear form, but changes the basis $A, B, C, \cdots \rightarrow A+B, B, C, \ldots$. Thus, it preserves the nondegeneracy property of the adjacency matrix.

The subspace $\mathcal{F}_{1}$ is spanned by the graph $p_{1}$ with a single vertex (whence no edges), which is a primitive element. Since $\mathcal{F}$ is the polynomial ring in its primitive elements, each homogeneous space $\mathcal{F}_{n}$ admits a decomposition into the direct sum of two subspaces, one of which is the subspace of polynomials in primitive elements of degree greater than 1 , and the other one is the space of polynomials divisible by $p_{1}$. We define the framed Conway graph invariant as the only multiplicative 4-invariant with values in the polynomial ring $\mathbb{C}[c, y]$ whose value on $p_{1}$ is $c$, and on the projection of any graph to the subspace of $p_{1}$-independent polynomials along the subspace of $p_{1}$-divisible polynomials coincides with the Conway graph invariant of the graph.

The values of the framed Conway graph invariant can be computed recursively. Take a graph $G$ and consider its projection to the subspace of graphs divisible by $p_{1}$. On this projection, the framed Conway graph invariant can be computed because of its multiplicativity. Now add to the result the value of the (unframed) Conway graph invariant on the graph. Now we can refine the statement of Theorem 5.

Theorem 6 The $\mathfrak{g l}(1 \mid 1)$ universal weight system is the pullback of the framed Conway graph invariant to chord diagrams under the homomorphism $\gamma$.

Proof The proof follows from two statements in [10]. Theorem 3.6 there states that setting $c=0$ in the value of $W_{\mathfrak{g r}(1 \mid 1)}$ on a chord diagram we obtain the result of deframing this weight system. Theorem 4.4 asserts that this value is exactly the Conway invariant of the chord diagram. The latter coincides with the Conway graph invariant of the intersection graph of the chord diagrams defined above. Since the deframing for chord diagrams is a pullback of the deframing for graphs, we are done.

\subsection{Ohtsuki's proof of Theorem 3}

In this section, we reproduce the proof of Theorem 3 due to TOhtsuki (private communication). The proof uses the algebras $\mathcal{A}(\uparrow \uparrow), \mathcal{C}(\uparrow \uparrow)$, and $\mathcal{B}(\boldsymbol{x}, \boldsymbol{y})$. We shall use the terminology and notation of Chmutov, Duzhin and Mostovoy [6] and refer the reader to this book for their precise definitions and properties. Here $\mathcal{A}(\uparrow \uparrow)$ is the algebra of chord diagrams supported on two vertical arrows $\uparrow \uparrow$ modulo the 4T-relation, $\mathcal{C}(\uparrow \uparrow)$ is the algebra of closed Jacobi diagrams on $\uparrow \uparrow$ modulo the AS, IHX, and STU relations, and $\mathcal{B}(\boldsymbol{x}, \boldsymbol{y})$ is the algebra of open Jacobi diagrams with univalent vertices labeled 
by $\boldsymbol{x}$ and $\boldsymbol{y}$ modulo the AS, IHX, and the link relations. All the three algebras are isomorphic to one another [6].

For each of these algebras and for a Lie (super)algebra $\mathfrak{g}$ with an invariant scalar product, one can define a universal weight system $W_{\mathfrak{g}}$ which takes values in $(U(\mathfrak{g}) \otimes U(\mathfrak{g}))^{\mathfrak{g}}$ in the case of the algebras $\mathcal{A}(\uparrow \uparrow)$ and $\mathcal{C}(\uparrow \uparrow)$, and in $(S(\mathfrak{g}) \otimes S(\mathfrak{g}))^{\mathfrak{g}}$ in the case of the algebra $\mathcal{B}(\boldsymbol{x}, \boldsymbol{y})$. Here $S(\mathfrak{g})$ denotes the symmetric tensor algebra of the vector space $\mathfrak{g}$. But according to the Poincaré-Birkhoff-Witt theorem, the vector spaces $U(\mathfrak{g})$ and $S(\mathfrak{g})$ are isomorphic. Therefore, we may think that the universal weight system $W_{\mathfrak{g}}$ takes values in $(U(\mathfrak{g}) \otimes U(\mathfrak{g}))^{\mathfrak{g}}$ for all the three algebras.

Theorem 3 would follow from the symmetry of the image of a chord diagram on $\uparrow \uparrow$ as we insert it into a chord diagram on a circle and take the universal weight system $W_{\mathfrak{g}}$ with values in $U(\mathfrak{g})^{\mathfrak{g}}$.

The $\mathfrak{g}=\mathfrak{s l}(2)$ case Here $\left(U(\mathfrak{s l}(2)) \otimes U(\mathfrak{s l}(2))^{\mathfrak{s l}(2)}\right.$ is generated by the three elements $c \otimes 1,1 \otimes c$, and

$$
W_{\mathfrak{s l}(2)}(\hat{\uparrow} \hat{\uparrow}),
$$

where $c$ is the Casimir element in $U(\mathfrak{s l}(2))$. Obviously, after inserting them into a chord diagram on a circle they become symmetric.

The $\mathfrak{g}=\mathfrak{g l}(1 \mid 1)$ case The universal weight system $W_{\mathfrak{g l}(1 \mid 1)}$ vanishes on any Jacobi diagram containing either of the fragments

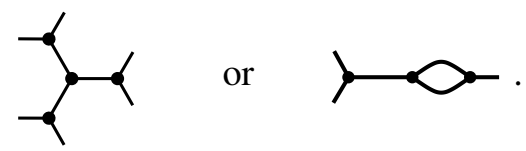

The quotient space of $\mathcal{B}(\boldsymbol{x}, \boldsymbol{y})$ modulo Jacobi diagrams with these fragments is generated by the diagrams

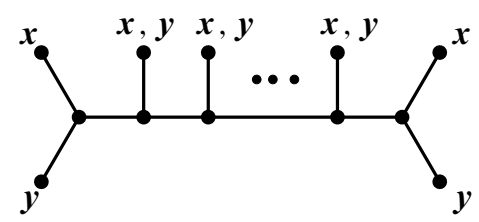

and

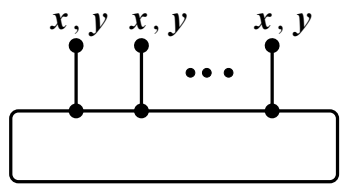

where the notation $\boldsymbol{x}, \boldsymbol{y}$ means that the corresponding univalent vertices are labeled either by $\boldsymbol{x}$ or by $\boldsymbol{y}$. These diagrams become symmetric after the insertion into a chord diagram on the circle modulo the above mentioned diagrams.

This completes Ohtsuki's proof. 


\section{References}

[1] D Bar-Natan, The Knot Atlas, http: //www .math.toronto.edu/drorbn/KAtlas/

[2] D Bar-Natan, On the Vassiliev knot invariants, Topology 34 (1995) 423-472 MR1318886

[3] A Bouchet, Reducing prime graphs and recognizing circle graphs, Combinatorica 7 (1987) 243-254 MR918395

[4] A Bouchet, Circle graph obstructions, J. Combin. Theory Ser. B 60 (1994) 107-144 MR1256586

[5] S V Chmutov, S V Duzhin, S K Lando, Vassiliev knot invariants I: Introduction, from: "Singularities and bifurcations", Adv. Soviet Math. 21, Amer. Math. Soc., Providence, RI (1994) 117-126 MR1310597

[6] S V Chmutov, S V Duzhin, J Mostovoy, CDBooK. Introduction to Vassiliev Knot invariants (2007) a preliminary draft version of a book about Chord Diagrams Available at http://www.math.ohio-state.edu/ chmutov/preprints/ cdbook-feb07.pdf

[7] S V Chmutov, A N Varchenko, Remarks on the Vassiliev knot invariants coming from $\mathrm{sl}_{2}$, Topology 36 (1997) 153-178 MR1410469

[8] B Courcelle, Circle graphs and Monadic Second-order logic, preprint (2005) Available at http://www.labri.fr/perso/courcell/ArticlesEnCours/ CircleGraphsSubmitted.pdf

[9] W H Cunningham, Decomposition of directed graphs, SIAM J. Algebraic Discrete Methods 3 (1982) 214-228 MR655562

[10] J M Figueroa-O'Farrill, T Kimura, A Vaintrob, The universal Vassiliev invariant for the Lie superalgebra gl(1|1), Comm. Math. Phys. 185 (1997) 93-127 MR1463035

[11] C P Gabor, K J Supowit, W L Hsu, Recognizing circle graphs in polynomial time, J. Assoc. Comput. Mach. 36 (1989) 435-473 MR1072233

[12] M Kontsevich, Vassiliev's knot invariants, from: “I. M. Gel'fand Seminar”, Adv. Soviet Math. 16, Amer. Math. Soc., Providence, RI (1993) 137-150 MR1237836

[13] S K Lando, On a Hopf algebra in graph theory, J. Combin. Theory Ser. B 80 (2000) 104-121 MR1778203

[14] S K Lando, A K Zvonkin, Graphs on surfaces and their applications, Encyclopaedia of Mathematical Sciences 141, Springer, Berlin (2004) MR2036721With an appendix by Don B. Zagier, Low-Dimensional Topology, II

[15] T Q T Le, J Murakami, The universal Vassiliev-Kontsevich invariant for framed oriented links, Compositio Math. 102 (1996) 41-64 MR1394520

[16] B Mellor, Intersection graphs for string links, J. Knot Theory Ramifications 15 (2006) 53-72 MR2204497 
[17] H R Morton, P R Cromwell, Distinguishing mutants by knot polynomials, J. Knot Theory Ramifications 5 (1996) 225-238 MR1395780

[18] J Murakami, Finite type invariants detecting the mutant knots, from: "Knot Theory. A volume dedicated to Professor Kunio Murasugi for his 70th birthday", (M Sakuma, et al, editors) (2000)

[19] C Soulié, Complete invariant graphs of alternating knots arXiv:math.CO/0404490

[20] A Vaintrob, Vassiliev knot invariants and Lie S-algebras, Math. Res. Lett. 1 (1994) 579-595 MR1295552

[21] D J A Welsh, Matroid theory, London Math. Soc. Monographs 8, Academic Press, London (1976) MR0427112

The Ohio State University - Mansfield

1680 University Drive, Mansfield OH 44906, USA

Institute for System Research RAS and the Poncelet Laboratory

Independent University of Moscow

Bolshoy Vlasyevskiy Pereulok 11, Moscow 119002, Russia

chmutov@math.ohio-state.edu, lando@mccme.ru

http://www.math.ohio-state.edu/ chmutov/, http://www.mccme.ru/ium/

$\sim$ lando/

Received: 16 May 2007 Revised: 14 September 2007 